\title{
De novo assembly of the pepper transcriptome (Capsicum annuum): a benchmark for in silico discovery of SNPs, SSRs and candidate genes
}

Hamid Ashrafi ${ }^{*}$, Theresa Hill1', Kevin Stoffel ${ }^{1}$, Alexander Kozik², JiQiang Yao ${ }^{1,3}$, Sebastian Reyes Chin-Wo ${ }^{1,2}$ and Allen Van Deynze ${ }^{1}$

\begin{abstract}
Background: Molecular breeding of pepper (Capsicum spp.) can be accelerated by developing DNA markers associated with transcriptomes in breeding germplasm. Before the advent of next generation sequencing (NGS) technologies, the majority of sequencing data were generated by the Sanger sequencing method. By leveraging Sanger EST data, we have generated a wealth of genetic information for pepper including thousands of SNPs and Single Position Polymorphic (SPP) markers. To complement and enhance these resources, we applied NGS to three pepper genotypes: Maor, Early Jalapeño and Criollo de Morelos-334 (CM334) to identify SNPs and SSRs in the assembly of these three genotypes.

Results: Two pepper transcriptome assemblies were developed with different purposes. The first reference sequence, assembled by CAP3 software, comprises 31,196 contigs from $>125,000$ Sanger-EST sequences that were mainly derived from a Korean $F_{1}$-hybrid line, Bukang. Overlapping probes were designed for 30,815 unigenes to construct a pepper Affymetrix GeneChip ${ }^{\circledR}$ microarray for whole genome analyses. In addition, custom Python scripts were used to identify 4,236 SNPs in contigs of the assembly. A total of 2,489 simple sequence repeats (SSRs) were identified from the assembly, and primers were designed for the SSRs. Annotation of contigs using Blast2GO software resulted in information for $60 \%$ of the unigenes in the assembly. The second transcriptome assembly was constructed from more than 200 million Illumina Genome Analyzer II reads (80-120 nt) using a combination of Velvet, CLC workbench and CAP3 software packages. BWA, SAMtools and in-house Perl scripts were used to identify SNPs among three pepper genotypes. The SNPs were filtered to be at least $50 \mathrm{bp}$ from any intron-exon junctions as well as flanking SNPs. More than 22,000 high-quality putative SNPs were identified. Using the MISA software, 10,398 SSR markers were also identified within the Illumina transcriptome assembly and primers were designed for the identified markers. The assembly was annotated by Blast2GO and 14,740 (12\%) of annotated contigs were associated with functional proteins.

Conclusions: Before availability of pepper genome sequence, assembling transcriptomes of this economically important crop was required to generate thousands of high-quality molecular markers that could be used in breeding programs. In order to have a better understanding of the assembled sequences and to identify candidate genes underlying QTLs, we annotated the contigs of Sanger-EST and Illumina transcriptome assemblies. These and other information have been curated in a database that we have dedicated for pepper project.
\end{abstract}

Keywords: Pepper, Capsicum spp, Molecular Markers, EST, Transcriptome, RNAseq, Annotation, SNP, SSR, SPP

\footnotetext{
*Correspondence: ashrafi@ucdavis.edu

${ }^{1}$ Seed Biotechnology Center, University of California, Davis, 1 Shields Ave, Davis, CA 95616, USA

Full list of author information is available at the end of the article
} 


\section{Background}

Pepper (Capsicum spp.) is a member of the Solanaceae family which is becoming an increasingly important vegetable crop worldwide due to its wide diversity and high quality in flavor, concentration of vitamins and other antioxidants. In addition to its dietary and culinary importance, capsaicinoid compounds of pepper are being used in the pharmaceutical industry [1]. Pepper has five domesticated and 15-20 wild crossable species. Similar to other members of the Solanaceae family such as tomato, pepper has been used as a model organism for classical and molecular genetics analyses. Selfing and crosses among and within certain species can be made readily and multiple generations can be easily produced each year. This creates the genetic diversity that is required for conventional breeding programs. However, the commercial application of these genomic resources for gene discovery and molecular breeding has been limited by paucity of available informative molecular markers. The limited amount of molecular markers is primarily due to lack of availability of the pepper genome sequence and sequence resources. For instance, over the past two decades, a variety of molecular markers (AFLP, RFLP, SSR, COSII and RAPD) have been developed and applied to several intra- and inter-specific crosses of pepper [2-4]. However, due to their nature, the majority of these markers, except COSII and tomato cDNA markers, that have been used in the published genetic maps of pepper are not high throughput or gene-based.

From among many types of molecular markers that have been developed during the past three decades, Simple Sequence Repeats (SSRs) and Single Nucleotide Polymorphisms (SNPs) are the most attractive ones for breeding [5]. SSRs are co-dominant and can be assayed in any laboratory with minimum facilities or they can be automated with capillary sequencers for moderate throughput. On the other hand, SNPs are extremely abundant; the majority are biallelic; they are easily scored and can be tightly linked to or are the actual cause of allelic (phenotypic) differences in traits. Moreover, there are several high-throughput technologies based on allele-specific PCR, hybridization and single base-pair extension which makes them cost-effective for assaying large numbers of genotypes once robust SNPs have been identified. However, without using recent bioinformatics tools and next generation sequencing (NGS), identifying SNPs and SSRs within a genome as large as pepper (3.5 Gb) [6] is not a trivial task.

Prior to the advent of NGS technologies, discovering putative SNPs was achieved using low throughput electrophoresis or capillary sequencing $[7,8]$. These methods are lengthy, low coverage and expensive per data point. However, we should recognize that Sanger sequencing has provided a wealth of EST sequences that have been the primary basis of identifying SNPs [9]. In 2006 at the onset of the Pepper GeneChip ${ }^{\circledR}$ project the sequencing resources of pepper were largely limited to the EST sequences that were developed by Dr. Doil Choi at Seoul National University. Assembling the EST sequences into unigenes and mining SNPs in silico is one of the approaches that has been used for marker development $[7,10]$. In order to take the genotyping resources of pepper to the next level- we used Affymetrix GeneChip arrays $[11,12]$ as a new tool for massively parallel marker discovery and genotyping in pepper. This novel tool uses a new generation of markers called Single Position Polymorphisms or SPPs [12]. Therefore, assembling ESTs enabled us, first to design and produce the genotyping chips and second to extract a wealth of polymorphism in pepper.

In recent years, sequencing of expressed genes (transcriptomes) using NGS technologies such as SOLiD, Illumina and 454, has been used for gene discovery and allele mining [13-15]. This method, also known as RNAseq, has been used in many plant and animal species such as maize [13], brassica [14], Arabidopsis [16], rice [17], human [18], and mouse [19]. With the advent of NGS technologies, the number of publications describing de novo assemblies of plants transcripts and other organisms has been increasing constantly. In addition to availability of sequences, bioinformatics tools have also been developed to process, analyze and store the massive data that are generated daily. For instance, one of the most popular assemblers is the Velvet [20] software package which is able to assemble short reads derived from Illumina into contigs using de Bruijn graphs [20] algorithm. However, Velvet is not the only assembler for short reads. SOAP de novo, ABySS [21] and CLC Genomics Workbench, which is commercially available, are just a few examples of many other assemblers. In the current study we took advantage of both Velvet and CLC to make de novo assemblies of transcriptomes of three pepper lines, Maor, Early Jalapeño and CM334.

The final goal in many transcriptome sequencing efforts is to annotate sequences by connecting them to biological information. Annotation of sequences allows one to have insight into the function and structure of the genome. Without annotation, sequences have little meaning. Availability of intronic regions through genome sequencing facilitates gene model predictions, which help to identify locations of regulatory elements as well as alternate splicing events. However, for pepper, a whole genome sequence is still not available and todate all annotations have been carried out on transcriptome sequences [22-24]. Automated annotation is an approach that provides us an immediate response to a question that we pose. Is there any similarity between 
unknown sequences and previously characterized sequences from the same or other species? Normally this will be done by the basic local alignment search tool (BLAST) to find the best matches between the unknown and known sequences followed by mapping the results to Gene Ontology (GO) terms [25] and associating the $\mathrm{GO}$ terms with functional proteins, using the results of previous steps. In the present study we performed an in silico annotation of both Sanger-EST and IGA transcriptome assemblies of pepper. The current annotation information can be used for candidate gene discovery, identification of regulatory elements and gene prediction before the full annotation of a pepper genome becomes available. We have also developed a MySQL database and a web interface that can be queried to find information about the assemblies, such as SSR or SNP makers within each contig and to find their corresponding annotation.

\section{Results}

\section{Pepper Sanger-ESTs assembly}

We developed a non-redundant set of unigenes based on all available sequences for pepper (in 2006) to design a tiling Affymetrix GeneChip array for marker discovery and application in pepper [11]. Merging the KRIBB (see list of URLs) sequences $(115,787)$ with the processed GenBank sequences $(9,905)$ resulted in 125,692 sequences. After trimming, a total of 123,489 sequences remained, including 121,867 EST sequences, 515 assembled mRNAs, 465 genomic sequences and 642 COSII marker sequences (Table 1). C. annuum made up 99.5\% of the sequences with minor representation from, C. frutescens, C. chinense and C. baccatum. Hereafter, the assembly of Sanger ESTs is called the Sanger-EST assembly. In the Sanger-EST assembly, 32,071 unigenes were obtained with 12,970 consensus sequences and 19,101 singletons. The number of unigenes account for $25.8 \%$ of initial input sequences $(123,489)$. Unigenes with a size less than 200 nucleotides (nt) accounted for $2.7 \%$ of the total unigenes. The summary statistics of the Sanger-EST assembly are presented in Figure 1a and Table 2. The final assembly, consisting of 31,196 unigenes greater than 200 nt, was annotated and mined for SSRs and SNPs.

\section{De novo pepper Illumina transcriptome assembly}

The Illumina transcriptome sequencing generated $\sim 53$ $\mathrm{M}, 57 \mathrm{M}$ and $90 \mathrm{M}$ cleaned and trimmed reads in CM334, Maor and Early Jalapeño, respectively. The raw data were submitted to NCBI Sequence Read Archive (SRA) under accession No. SRA052314.2 and the trimmed reads submitted to European Nucleotide Archive (ENA) under study number ERP001411. The more stringently trimmed reads ranged between 25 to $70 \mathrm{nt}$ in length as described in methods. To compare assembly
Table 1 A summary of sequences included in the pepper Sanger-EST assembly

\begin{tabular}{lll}
\hline $\begin{array}{l}\text { Sequence Type/ } \\
\text { Species }\end{array}$ & $\begin{array}{l}\text { No. of Sequences Before } \\
\text { Filtering }\end{array}$ & $\begin{array}{l}\text { Total on the } \\
\text { Chip }\end{array}$ \\
\hline ESTs & 125,320 & 123,489 \\
\hline C. annuum & 372 & \\
\hline C. chinense & & 465 \\
\hline Genomic & 318 & \\
\hline C. annuum & 28 & \\
\hline C. baccatum & 31 & \\
\hline C. chinense & 27 & \\
\hline C. frutescens & 61 & \\
\hline Others & & \\
\hline Annotated mRNA & 427 & \\
\hline C. annuum & 76 & \\
\hline C. chinense & 12 & \\
\hline Others & 642 & \\
\hline COSII & & \\
\hline
\end{tabular}

performance of different $k$-mer (see below for definition) values [20], we tested $k$ values of 31,35 and 41 bp. Applying different $k$-mers resulted in the use of different numbers of reads but the overall trend was toward the use of more reads in the assembly as the $k$-mer increased from 31 to 41. In Velvet, 64\% - 79\% of the sequences were used in each assembly as the $k$-mer value was increased. Both Velvet and CLC produced significantly fewer contigs, with average reductions ranging from $48 \%$ in Velvet to $35 \%$ in CLC, when using stringently trimmed data. For instance, in the case of Early Jalapeño by using untrimmed and trimmed data at $k=31 \mathrm{bp}$, the number of contigs generated in the two assemblies was 68,737 and 39,956, respectively. The fraction of contigs longer than $1 \mathrm{~KB}$ varied from $83 \%$ ( $k=31 \mathrm{bp})$ to $72 \%(k=31)$ for untrimmed and trimmed data (Table 3$)$. Median weighted (N50) lengths of assemblies were highest at $k=41 \mathrm{bp}$ for both untrimmed and trimmed data (Table 3). The meta assembly which is called hereafter the pepper IGA (Illumina Genome Analyzer) transcriptome assembly, comprises assembly of contigs from Velvet and CLC and had the largest median of all assemblies $(\mathrm{N} 50=1,647)$ with 123,261 contigs and an assembly of $>135 \mathrm{M}$ bases (Figure $1 \mathrm{~b}$ and Table 2 ). The final results and steps to generate de novo assembly of pepper IGA reads are presented in Table 4.

\section{Annotation of Sanger-EST assembly}

Both assemblies were annotated using Blast2GO software [26]. Blast2GO annotation is Gene Ontology (GO) based data mining for sequences with unknown function $[25,26]$. The results of each step of Blast2GO annotation of the Sanger-EST assembly are summarized in 


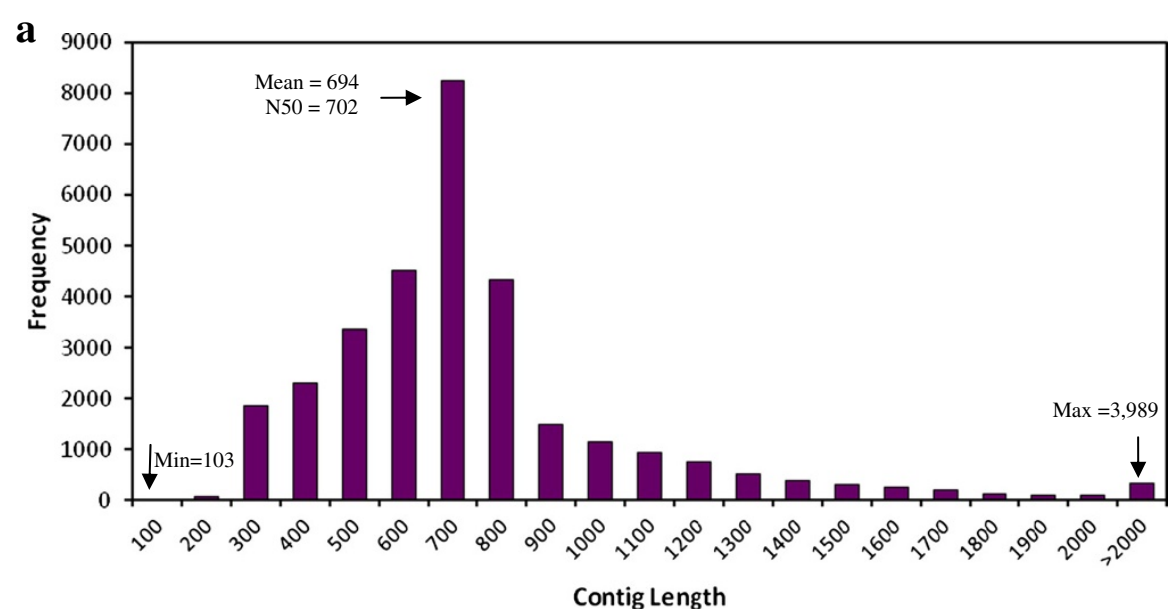

b

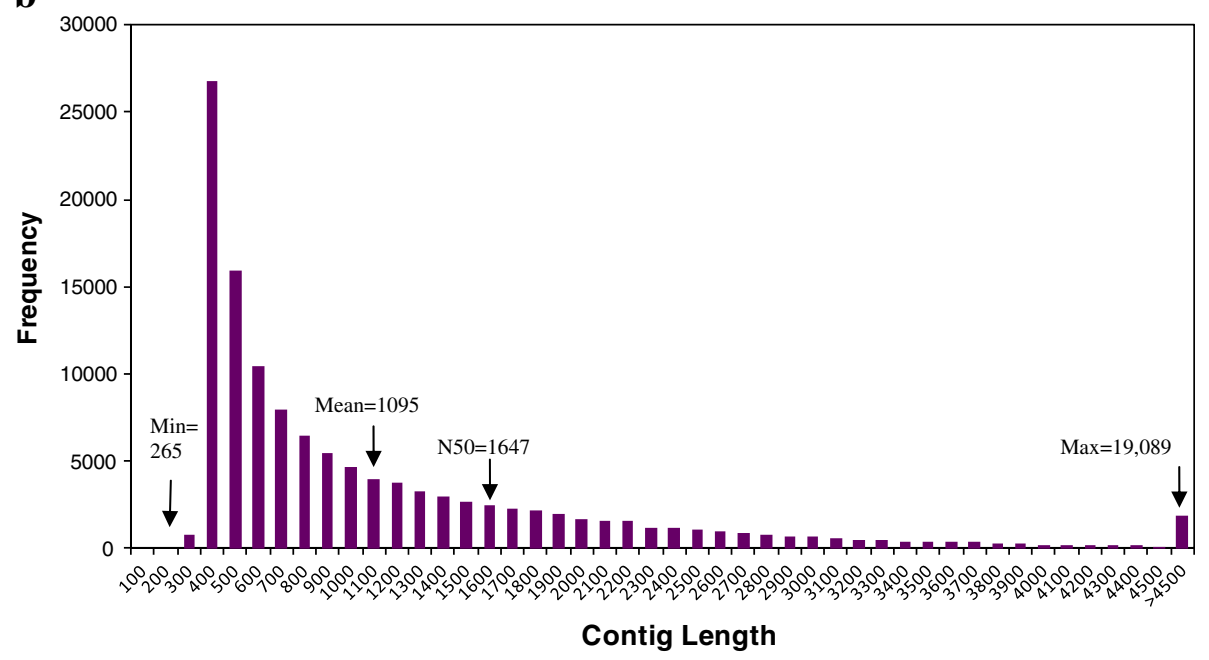

Figure 1 a) Distribution of contigs length in a) pepper Sanger-EST assembly b) distribution of contigs length in pepper IGA transcriptome assembly.

Figure 2a. BLASTX of the Sanger-EST assembly unigenes against the GenBank non-redundant protein database resulted in the identification of 24,003 (76.9\%) sequences with at least one significant alignment to an existing gene model and with an average contig length of 745 nt. These contigs covered $21.6 \mathrm{M}$ bases $(82.5 \%)$ of the total Sanger-EST assembly. The 7,193 unigenes that did not have any hit in the GenBank were on average $525 \mathrm{nt}$ long and were covering 3.8M (17.5\%) bases. The mapping step of Blast2GO resulted in association of $22,728(72.8 \%)$ unigenes with GO terms [25] (Figure 2a). The unigenes were assigned between 1 and $50 \mathrm{GO}$ terms with a weighted average of five GO terms per unigenes. The annotation step of Blast2GO assigned functions to $18,715(60 \%)$ of unigenes. A query with InterProScan increased the number of annotated unigenes by $17 \%$. The results of the Blast2GO annotation were merged with the results of the InterPro annotation to maximize the number of annotated sequences. By categorizing all BLASTX results, Vitis vinifera, Glycine max, Arabidopsis thaliana, Populus trichocarpa and Oryza sativa were among the top five plant species in terms of the total number of hits to the Sanger-EST unigenes (Figure 3a). However, when the results were categorized based on the highest similarity between each of the Sanger-EST unigenes and sequences in the databases, the top five plant species were $V$. vinifera, $P$. trichocarpa, Ricinus communis, G. max and Solanum lycopersicum (Figure 3b). Direct GO count graphs were created to categorize the sequences to several groups based on their biological processes, molecular functions and cellular component ontologies. Inside the biological processes category, sequences in cellular process, metabolic process, response to stimulus, biological regulation and localization had the highest frequencies. In terms of molecular function, transferase activity, nucleotide binding 
Table 2 Comparison of assembly of pepper Sanger ESTs versus assembly of IGA reads

\begin{tabular}{lrr}
\hline Statistics & \multicolumn{1}{c}{$\begin{array}{c}\text { EST } \\
\text { Assembly }\end{array}$} & \multicolumn{1}{c}{$\begin{array}{c}\text { Transcriptome } \\
\text { Assembly }\end{array}$} \\
\hline Number of Unigenes & 31,196 & 123,261 \\
\hline Total assembled nucleotides & $21,665,127$ & $135,019,787$ \\
\hline Average GC content (\%) & 41 & 39 \\
\hline Longest Contig Length & 3,989 & 19,089 \\
\hline Average Contig Length & 694.5 & 1,095 \\
\hline Median Contig Length & 651 & 697 \\
\hline N50 & 702 & 1,647 \\
\hline Number/Percent Contigs & 27,248 & 78,433 \\
Size $<1$ KB & & 27,436 \\
\hline $1-2$ KB & 3,634 & 10,616 \\
\hline $2-3$ KB & 288 & 3,955 \\
\hline $3-4$ KB & 26 & 1,559 \\
\hline $4-5$ KB & 0 & 1,184 \\
\hline $5-10 ~ K B$ & 0 & 78 \\
\hline $10-20 ~ K B$ & 0 &
\end{tabular}

and ion binding related sequences were the top three GO terms in the Sanger-EST assembly. Among cellular components, the GO terms corresponding to constituents of the cytosol (intracellular fluid), intracellular part (any constituent part of the living contents of a cell), plasma membrane and organelle had the highest numbers in the assembly [See Additional File 1: Figure S11a-c]. The results of annotation can be accessed and queried through the pepper GeneChip database [See list of URLs] or [See Additional File 2: Table S1].

\section{Annotation of IGA transcriptome assembly}

The three steps of Blast2GO annotation of the IGA transcriptome assembly are summarized in Figure 2b. A total of 63,202 contigs (51.3\%) with an average length of 1,495 nucleotides had at least one significant alignment with a protein in the non-redundant database of GenBank. These contigs covered $94.5 \mathrm{M}$ bases, $(70 \%)$ of the total assembly. However, the 60,055 (48.7\%) contigs that did not have hit to any sequence in GenBank were on average 674 nucleotide long and covered $40.5 \mathrm{M}$ bases, $(30 \%)$ of the total assembly. The mapping step of Blast2GO identified 37,918 (30.7\%) contigs with GO terms. A significant amount of mapping data (91.5\% of contigs with mapping information) were derived from UniProtKB database followed by TAIR and GR_protein. In addition, 13 other databases were searched but did not significantly contribute to the mapping process. Between 1-80 GO terms were assigned per sequence with a weighted average of $5 \mathrm{GO}$ terms per contig (Figure $2 \mathrm{~b}$ ). Twelve percent, $(14,740)$ of contigs, were annotated as functional proteins. The frequency of $\mathrm{GO}$ terms for shorter sequences $(<2.5 \mathrm{~KB})$ was less than that of longer sequences. The percentage of annotated sequences increased proportionally with their length, such that sequences longer than $4.8 \mathrm{~KB}$ were $100 \%$ annotated. As expected, the majority of annotations were inferred electronically compared to direct assays (14\%) [See Additional File 1: Figure S5-S6]. By counting all significant hits in the BLASTX result table, $V$. vinifera, $A$. thaliana and $O$. sativa were the top three species in terms of hit number (Figure 3c). As Figure 4c depicts, based on this grouping Solanum $s p$. did not have as many hits as other less closely related species to pepper. However, when we filtered the BLASTX results based on similarity of pepper contigs with Solanum species, Solanum sp. were ranked after $V$. vinifera, $P$. trichocarpa and $R$. communis (Figure 3d). InterProScan, Annex, and GO annotation query through more than 16 databases significantly increased annotation by $15 \%$. Direct GO count graphs were created to categorize the sequences based on their biological processes and molecular functions as well as their cellular component. Based on their biological processes sequences involved in cellular process, metabolic process and response to stimulus had the maximum frequencies. In terms of molecular function, nucleic acid binding elements comprised the highest numbers of

Table 3 The effect of trimming the reads and k-mer length on the number of contigs and N50 in IGA transcriptome assembly

\begin{tabular}{|c|c|c|c|c|c|c|c|c|c|c|c|c|}
\hline \multirow{2}{*}{$\begin{array}{l}\text { Type of Reads/ } \\
\text { k-mer value }\end{array}$} & \multicolumn{4}{|c|}{ CM334 } & \multicolumn{4}{|c|}{ Maor } & \multicolumn{4}{|c|}{ Early Jalapeño } \\
\hline & $\begin{array}{l}\text { Total } \\
\text { No. of } \\
\text { Contigs }\end{array}$ & $\begin{array}{l}\text { No. } \\
\text { Contigs > } \\
1 \mathrm{~KB}\end{array}$ & $\begin{array}{c}\text { Percent } \\
\text { Contigs > } \\
1 \mathrm{~KB}\end{array}$ & N50 & $\begin{array}{l}\text { Total } \\
\text { No. of } \\
\text { Contigs }\end{array}$ & $\begin{array}{l}\text { No. } \\
\text { Contigs > } \\
1 \mathrm{~KB}\end{array}$ & $\begin{array}{c}\text { Percent } \\
\text { Contigs > } \\
1 \mathrm{~KB}\end{array}$ & N50 & $\begin{array}{l}\text { Total } \\
\text { No. of } \\
\text { Contigs }\end{array}$ & $\begin{array}{l}\text { No. } \\
\text { Contigs > } \\
1 \text { KB }\end{array}$ & $\begin{array}{c}\text { Percent } \\
\text { Contigs > } \\
1 \text { KB }\end{array}$ & N50 \\
\hline Untrimmed/K31 & 65,337 & 52,179 & 80 & 603 & 62,570 & 50,306 & 80 & 589 & 68,737 & 57,077 & 83 & 497 \\
\hline Untrimmed/K35 & 64,096 & 49,875 & 78 & 680 & 61,561 & 48,345 & 79 & 660 & 68,237 & 55,564 & 81 & 562 \\
\hline Untrimmed/K41 & 52,099 & 36,770 & 71 & 947 & 50,290 & 35,900 & 71 & 926 & 58,431 & 44,045 & 75 & 777 \\
\hline Trimmed 25-70/K31 & 42,310 & 30,628 & 72 & 864 & 36,173 & 24,871 & 69 & 995 & 39,956 & 28,711 & 72 & 870 \\
\hline Trimmed 25-70/K35 & 34,525 & 22,627 & 66 & 1,109 & 30,202 & 18,859 & 62 & 1,205 & 34,497 & 23,039 & 67 & 1,057 \\
\hline Trimmed 25-70/K41 & 27,439 & 16,728 & 61 & 1,239 & 26,885 & 16,660 & 62 & 1223 & 28,588 & 18,162 & 64 & 1,165 \\
\hline
\end{tabular}

* The untrimmed reads were between $40-80 \mathrm{nt}$ long. The same reads were trimmed by 5 and $10 \mathrm{nt}$ from $5^{\prime}$ an $3^{\prime}$ ends respectively to eliminate the possible sequencing errors at the beginning and at the end of each read. The numbers were selected arbitrarily but $\mathrm{K}=31$ is the Velvet recommended value. 
Table 4 Summary statistics of transcriptome assembly of three pepper lines using Velvet, CLC and CAP3 assemblers

\begin{tabular}{|c|c|c|c|c|c|c|c|c|c|c|}
\hline \multirow[t]{2}{*}{ Name of Assembly } & \multirow[t]{2}{*}{ Assembler } & \multicolumn{3}{|c|}{ CM334 } & \multicolumn{3}{|c|}{ Maor } & \multicolumn{3}{|c|}{ Early Jalapeño (EJ) } \\
\hline & & $\begin{array}{l}\text { No. of } \\
\text { Contigs }^{a}\end{array}$ & N50 & $\begin{array}{l}\text { Total } \\
\text { Assembled nt. }\end{array}$ & $\begin{array}{l}\text { No. of } \\
\text { Contigs }^{a}\end{array}$ & N50 & $\begin{array}{l}\text { Total } \\
\text { Assembled nt. }\end{array}$ & $\begin{array}{l}\text { No. of } \\
\text { Contigs }\end{array}$ & N50 & $\begin{array}{l}\text { Total } \\
\text { Assembled nt. }\end{array}$ \\
\hline \multirow[t]{2}{*}{ Super Assembly } & Velvet $^{b}$ & 75,853 & 1,287 & $71,903,681$ & 70,459 & 1,303 & $67,210,074$ & 81,973 & 1,198 & $73,865,962$ \\
\hline & $\mathrm{CLC}^{\mathrm{C}}$ & 83,187 & 1,357 & $79,564,926$ & 76,542 & 1,389 & $74,367,265$ & 81,528 & 1,347 & $78,144,374$ \\
\hline Mega Assembly & CAP3 & 83,113 & 1,488 & $84,792,180$ & 76,375 & 1,526 & $79,383,673$ & 82,614 & 1,488 & $84,973,865$ \\
\hline \multicolumn{8}{|l|}{ (Velvet+CLC) } & \multicolumn{3}{|c|}{ Combined CM334, Maor, EJ } \\
\hline Meta Assembly (3 lines) & CAP3 & & & & & & & 123,261 & 1,647 & $135,019,787$ \\
\hline
\end{tabular}

${ }^{a}$ No of contigs longer than 300 nucleotides were included in all assemblies.

${ }^{b}$ Six Velvet iterations including three k-mers of normally trimmed data and 3 k-mers of stringently trimmed data were assembled using CAP3 program.

'Two CLC iterations including one normally trimmed data and one stringently trimmed data were assembled using CAP3 program.

sequences in the IGA transcriptome assembly, followed by transferase activity and nucleotide binding related sequences. Cellular component constituents of intracellular organelle, cytoplasm and cytoplasmic part and plasma membrane were among sequences that had the maximum numbers in the assembly [See Additional File 1: Figure S12a-c]. The KEGG maps for more than 130 metabolic pathways were generated for both assemblies and the results were exported. Two examples of KEGG maps for the Pyrimidine metabolism pathway are depicted in Figure 4a-b. The KEGG map files, the Blast2GO project files (.dat files), InterProScan and BLASTX files are available to download at the Pepper GeneChip website. The results of annotation also can be accessed
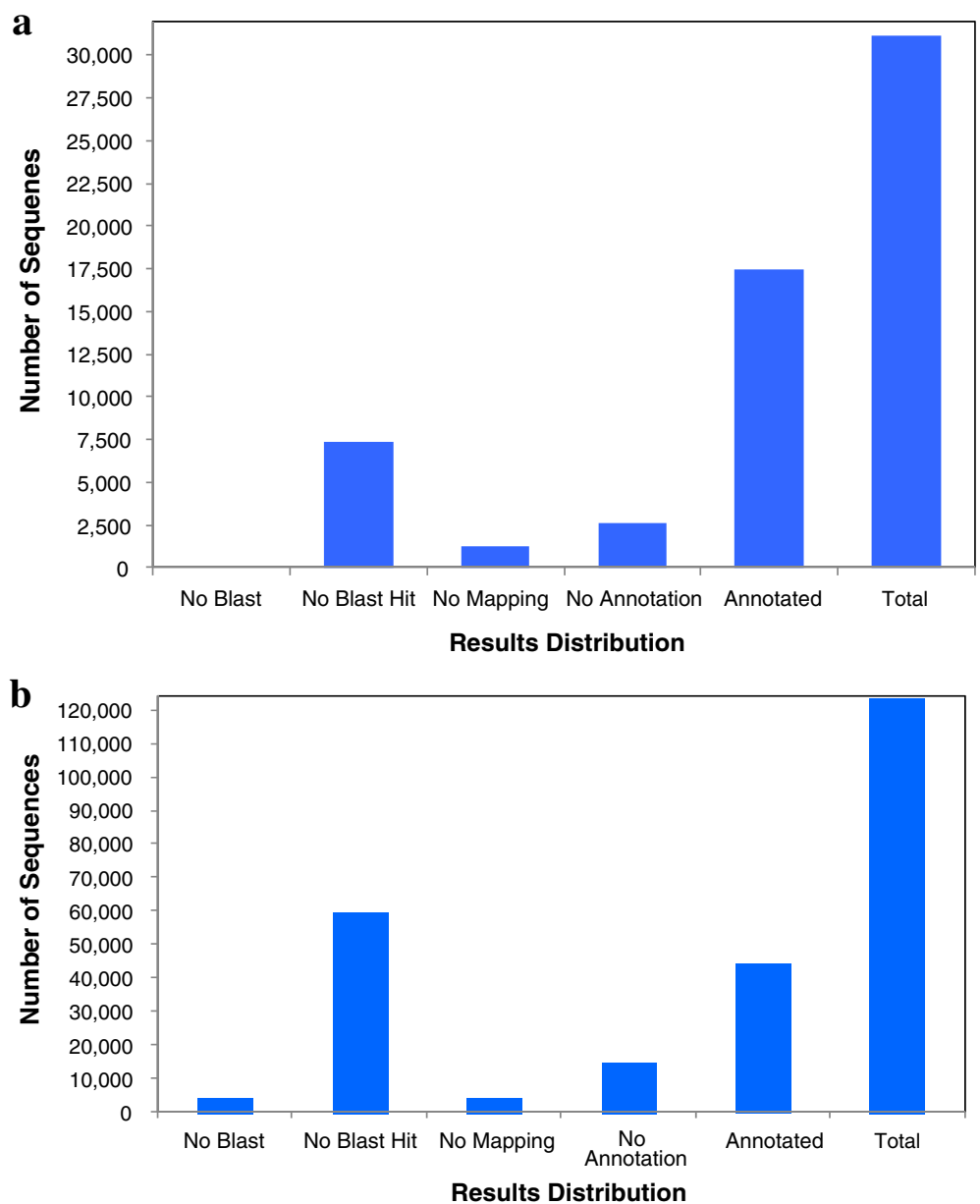

Figure 2 Distribution of Blast2GO three-step processes including BLASTX, mapping and annotation of for a) Sanger-EST assembly and b) IGA transcriptome assembly. 


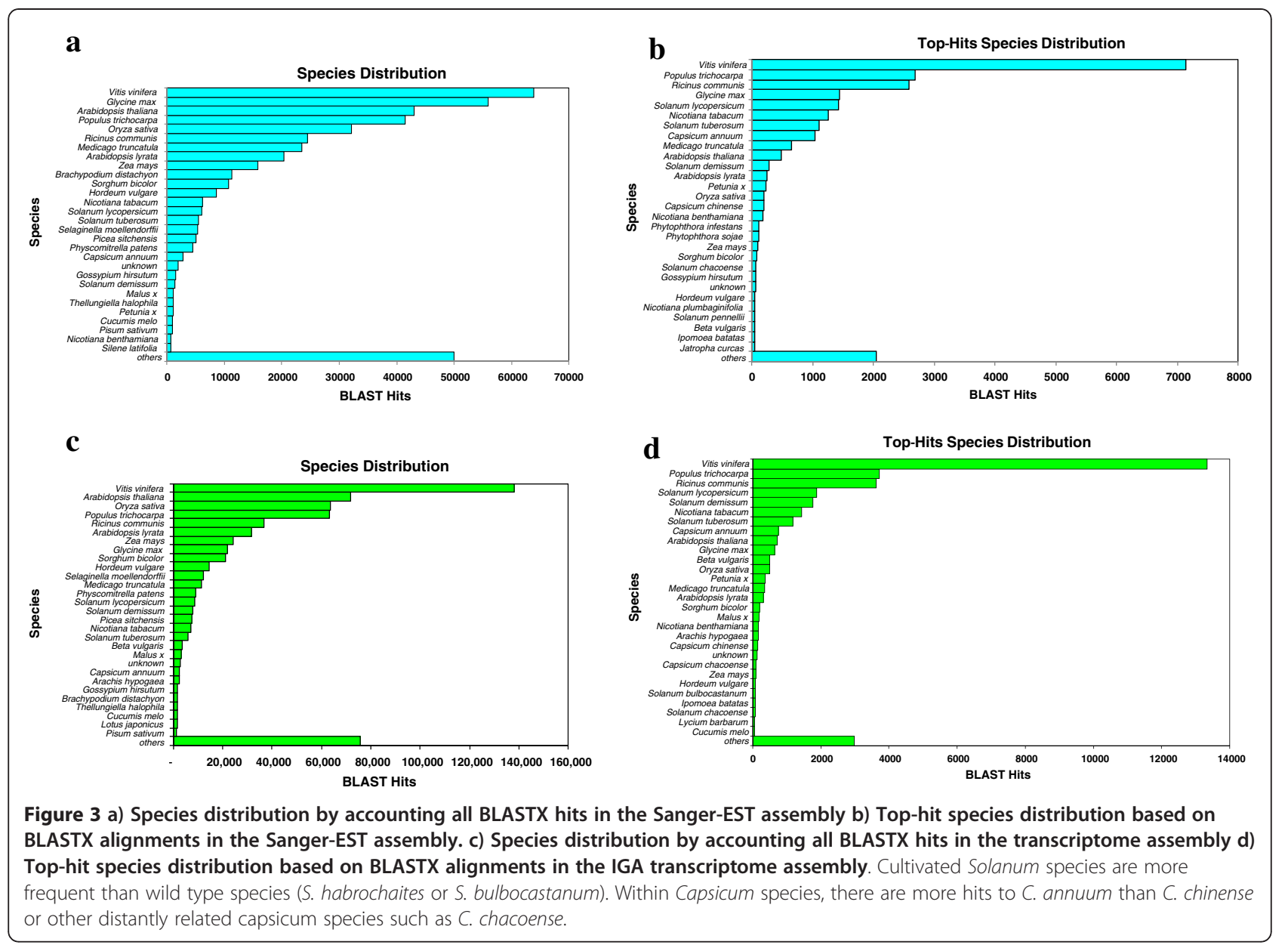

through the Pepper GeneChip database or [See Additional File 2: Table S2].

\section{SSR discovery in the Sanger-EST and the IGA transcriptome assemblies}

From the 31,196 unigenes (21.6 M bp) in the SangerEST assembly, 2,357 (7.5\%) unigenes contain putative SSRs, from which 253 unigenes bear more than one SSR marker signature. A total of 2,489 SSRs with simple repeats and 183 ( 7\%) SSRs with compound formation were identified. From 123,261 contigs that were examined in the IGA transcriptome assembly, 9,498 contigs were identified with 10,396 SSRs of which 617 (5.6\%) SSRs were of compound formation. From 9,498 SSR-containing contigs, $1,236(13.0 \%)$ had more than one SSR sequence. Using Primer3 software we were able to design primers for 1,533 and 7,458 putative SSR markers in the SangerEST and the IGA assemblies, respectively. A total of 859 SSRs were identified with identical motif and size between the two assemblies, resulting in 8,132 unique SSRs. In both assemblies, di-nucleotide AG/CT was the most frequent SSR motif followed by AC/GT or AT/TA. The tri-nucleotide motif AAC/GTT was more frequent in the IGA transcriptome assembly than that of the Sanger-EST assembly, while AAG/CTT was more frequent in the Sanger-EST assembly than the IGA transcriptome assembly. Overall, tri-nucleotide motifs were more frequent in our IGA transcriptome assembly than the Sanger-EST assembly. Longer motifs such as tetra and penta-nucleotide motifs were less frequent than diand tri- nucleotide motifs [See Additional File 3: Tables S3 and S6]. Additional File 3: Tables S4 and S7 provide lists of SSRs in the Sanger-EST and the IGA assemblies, respectively. Where possible the primers were designed for SSRs in both assemblies [See Additional File 3: Tables S5 and S8] and common SSRs are listed in Additional File 3: Table S9.

\section{SNP discovery in Sanger-EST assembly}

A majority of ESTs that were used in the Sanger-EST assembly were obtained from cDNA libraries of a Korean $\mathrm{F}_{1}$ hybrid (Bukang). Accordingly, most of the SNPs that we identified would belong to polymorphism between the parents of this particular $F_{1}$ individual. In the Sanger-EST assembly we had 12,970 unigenes that resulted from greater than one EST. The remaining 


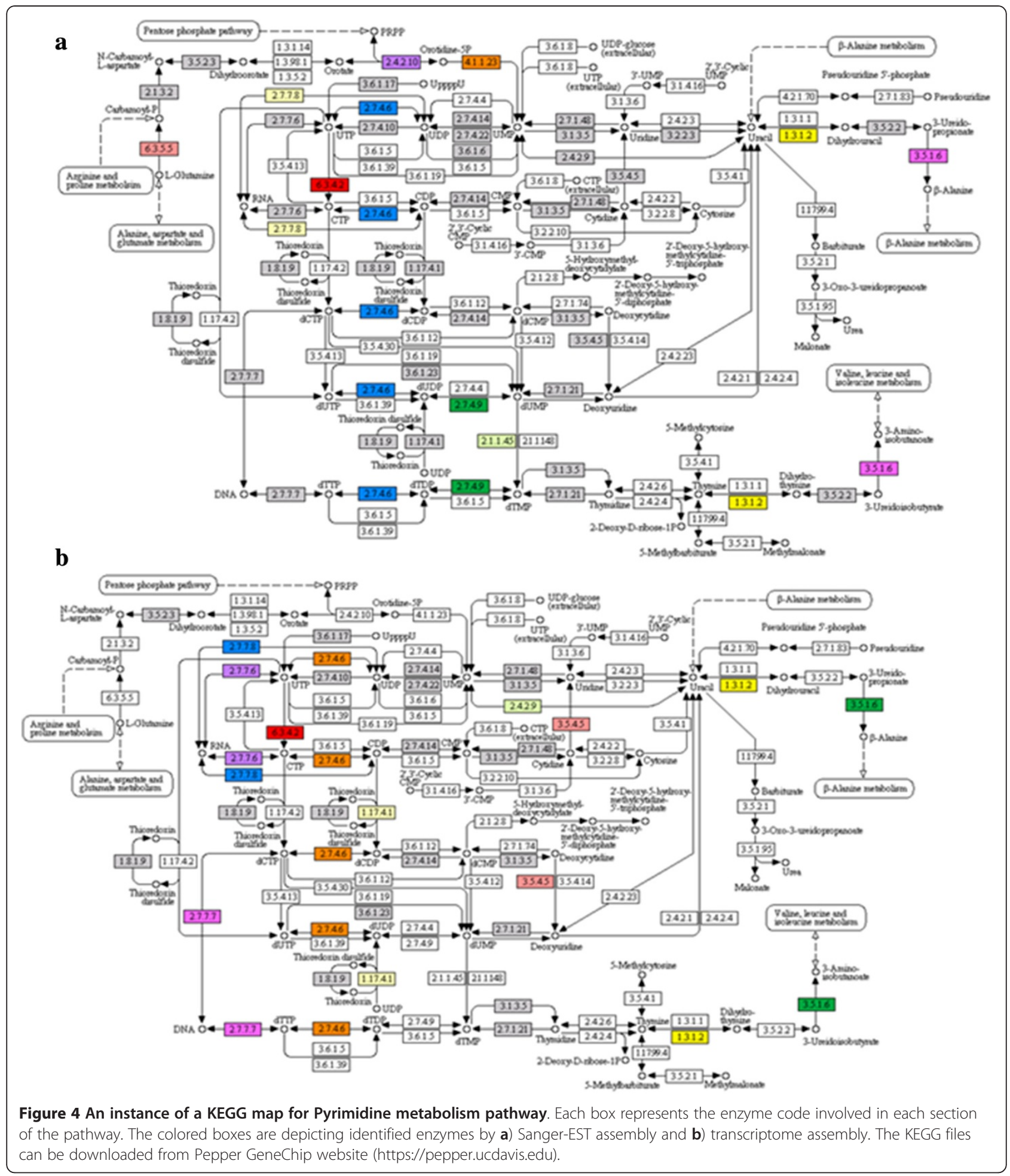

unigenes were single sequences that could not be examined for presence of SNPs. Analysis of 12,970 unigenes resulted in identification of 4,234 putative SNPs from 1,854 contigs [See Additional File 3: Table S10], an average of 0.3 SNPs per contig. The 12,970 contigs comprise $11,847 \mathrm{~KB}$ of pepper Sanger-EST assembly. Therefore, on average 1 SNP per 2,798 bases of pepper Sanger-EST assembly was identified.

\section{SNP discovery in the IGA transcriptome assembly}

The IGA transcriptome assembly comprised 123,261 unigenes including 48,642 contigs (assembled contigs of 
three genotypes) and 74,619 singletons (assembled sequences from a single genotype). In order to make a fair comparison between the IGA transcriptome assembly and the Sanger-EST assembly, we employed only 48,642 contigs of the IGA transcriptome assembly to discover SNPs. Using only contigs in the assembly a total of 47,686 putative SNPs were identified, that is $\sim 1$ SNP per contig. SNPs were filtered for the presence of an adjacent SNP in the vicinity of 50 bases. After filtering, a total of 30,495 SNPs were remaining that were used in calculation of SNP density. The 48,642 contigs that were mined for SNPs represented 76,952 KB of the pepper IGA transcriptomes that contains on average 1 SNP per 2,523 bases identified in the pepper IGA transcriptome assembly.

In order to provide a set of more reliable set of putative SNPs to the public; in a separate analysis all 123,261 sequences of IGA transcriptome assembly were used, thereby a total of 51,029 putative SNPs were identified. These putative SNPs were first filtered for the presence of flanking intronic region junction, adjacent putative SNPs as well as heterozygote positions in their 50 bp vicinity (See Materials and Methods). After applying all of the filters across the three genotypes used for the IGA transcriptome assembly, a total of 22,863 putative SNPs were retained [See Additional File 3: Table S11]. The SNPs were submitted to Database of Single Nucleotide Polymorphisms (dbSNP). Bethesda (MD): National Center for Biotechnology Information, National Library of Medicine. dbSNP accession:[ss523750580 - ss524344808], (dbSNP Build ID: B138), available from NCBI SNP database website.

\section{Comparison of SNPs between the Sanger-EST and the IGA transcriptome assemblies}

To identify the unique and common SNPs between the two assemblies, the following alignments were made reciprocally for each SNP using BLASTN. A 101 nt fragment from the Sanger-EST assembly with a SNP at the center was aligned with all SNP-containing fragments (101 nt) of the IGA transcriptome assembly and vice versa. A valid hit counted as the one with a minimum of $80 \mathrm{nt}$ matches in the alignment. The reciprocal comparison of SNPs from each assembly determined that 3,918 out of 4,235 SNPs were unique to the Sanger-EST assembly [See Additional File 3: Table S12]. Of the 22,863 SNPs from the IGA transcriptome assembly 22,548 (98.6\%) SNPs were unique to that assembly [See Additional File 3: Table S13]. Finally, a total of 316 common SNPs between the two assemblies were identified by this analysis [See Additional File 3: Table S14] resulting in 26,782 unique SNPs.

\section{SNP validation}

Out of 142 SNPs assayed, three (2.1\%) did not produce any PCR product and $13(9.1 \%)$ had ambiguous calls, that is one allele was called correctly according to our SNP discovery pipeline but the alternate allele could not be unequivocally determined by KASPar assay. Out of 126 remaining SNP assays [88 Sanger-EST assembly SNPs and 38 verified by SPPs (See Stoffel et al. for definition)], $113(89.7 \%)$ were polymorphic (at least one of the two alleles was observed across 47 genotypes) and 13 were monomorphic across the genotyping panel (FDR $=10 \%)$. From 113 polymorphic assays, 78 (70\%) and 35 (30\%) were SNPs and SPP, respectively. Therefore, 78 out of 88 (89\%) amplifiable SNP assays were polymorphic across the diversity panel described in Hill et al. [11] [See Additional File 3: Table S15]. We also investigated the polymorphism rate of 78 putative SNPs among the three pepper genotypes that were used for transcriptome assembly (CM334, EJ and Maor). A total of 40 out of $78(51 \%)$ assays were polymorphic in the diversity panel. However, 16 out of 40 SNPs identified in the IGA transcriptome assembly were called correctly based on KASPar assay. Although the remaining 24 putative SNPs showed polymorphism between lines, they had low coverage ( $<10$ reads) and did not meet our filtering criteria (at least 20 reads), therefore were not included in our final SNP dataset from the IGA transcriptome assembly.

\section{Discussion}

We report on two transcriptome assemblies of pepper, the first is based on Sanger-EST sequences was used in the pepper GeneChip ${ }^{\circledR}$ project [11]. The second is based on a collection of transcriptomes of three pepper lines that were sequenced by IGA technology. The majority of pepper EST sequences that were used in the current project had been first assembled by Kim et al. (2008), in which they had assembled 22,011 unigenes with an average consensus sequences length of 1,688 bp. However, in order to construct the pepper GeneChip microarray prior to the Kim publication, we added all pepper sequences and resources that were available at the time (2006) of the assembly. In addition to C. annuum EST sequences from Korean $\mathrm{F}_{1}$ hybrid of Bukang, we added $>700$ sequences from other $C$. annuum cultivars and other pepper species such as $C$. baccatum, C. frutescens and $C$. chinense. We added pepper genomic and mRNA sequences from GenBank and COS marker sequences from Solanaceae Genome Network (SGN) and UC Davis [7]. We used a combination of several in-house scripts and CAP3 to make our assembly, while Kim et al. took a different approach to make the assembly. Regardless of the methods used to assemble EST sequences, the database that Kim et al. has created is useful per se to query 
for the sequence information and find annotation of each contig. We have enhanced the information for Sanger pepper ESTs by mining and validating a subset of SNPs from this assembly. We have also leveraged the information to develop an Affymetrix tiling array to construct two ultra-saturated genetic maps of pepper [27] and to evaluate genetic diversity in pepper breeding germplasm [11]. Overall, we were able to map $>17,500$ unigenes representing over 3,000 genetic bins of pepper [27]. In the second pepper assembly we attempted to capture as many transcribed genes as possible by collecting tissues from three different genotypes (than Bukang) in different developmental stages. Recently a transcriptome assembly of two pepper parental lines (CM334 and Taean) and their hybrid line (TF68) was carried out by Lu et al. [22,23]. Lu et al. used the GS-454 FLX Titanium (Roche, Mannheim, Germany) to sequence mRNA that was collected from fruits of greenhouse-grown peppers. The pepper land race, CM334, in the $\mathrm{Lu}$ et al. study was the same land race that we used, but they sequenced it by Roche 454 system and sampled fewer tissues. Furthermore, we normalized our libraries prior to sequencing. Using GS de novo assembling software (Newbler) they were able to assemble 25,597 (N50=911), 29,335 (N50=898) and 33,530 (N50=884) contigs in each of CM334, Taean and TF68, respectively. Functional annotation of these contigs was performed by FunCat [28], by which it was determined that the majority of contigs were involved in proteins with binding function, regulation and metabolism. These results are similar to our functional annotation. The Capsicum transcriptome database, a most recent study of pepper transcriptomes, was recently introduced by Góngora-Castillo et al. [24]. Using Sanger and GS-pyrosequencing technologies they sequenced thirty-three cDNA libraries of $C$. annuum var. Sonora Anaheim and C. annuum var. Serrano Tampiqueño. Finally, creating a hybrid assembly of SangerEST sequences and GS-pyrosequencing using the 454 Newbler program was made using over $1.9 \mathrm{M} 454$ reads and Sanger-EST sequences. This assembly consists of 32,314 contigs with N50 of 631 and contig length ranging from 100-3,033 nt. The number of contigs of their assembly was close to our Sanger-EST assembly, as well as the three pepper assemblies reported by $\mathrm{Lu}$ et al. $[22,23]$. However, the number of contigs might be slightly over estimated because they took into account contigs with a minimum of $100 \mathrm{nt}$ in length, whereas in our Sanger-EST assembly the smallest contig was $200 \mathrm{nt}$.

While the 454 system generates long sequences, it suffers from low sequence depth, which is the unique advantage of the IGA system. Roche 454 performs poorly over the homopolymer regions of the genome. While IGA performs better on those regions, it has the disadvantage of generating short reads. Therefore a hybrid assembly of long and short reads to resolve the shortfalls of both sequencing systems would improve the quality of assembly. In spite of using IGA technology alone by sequencing three lines of pepper and boosting the number and length of reads (currently up to $120 \mathrm{nt}$ ) per IGA lane, we were able to assemble $>135 \mathrm{M}$ nucleotides in our assembly, which is 26 times more than any previously reported assemblies. In addition to the number of bases assembled, the N50 of the transcriptome assembly of this study is twice that of assemblies that were made with pyrosequencing alone.

In the present study we also annotated the two assemblies of pepper transcriptomes. According to the percentage of annotated contigs, $65 \%$ of the Sanger-EST assembly contigs and $35 \%$ of the IGA transcriptome assembly contigs were annotated. There are a number of reasons for the lower percentage of annotation of the IGA transcriptome assembly; one is that there were more novel sequences in the IGA transcriptome assembly compared to the Sanger-EST assembly. These new sequences did not have any hit in the GenBank, and as a result the number of sequences that were not annotated increased. Contig length also contributes to lower annotation. Since there were relatively more short contigs in the IGA transcriptome assembly than the Sanger-EST assembly, the percent of annotated sequences was lower. Also, during the Sanger sequencing procedure there is a cloning step involved in library construction, which favors selection for higher copy number transcripts, resulting in redundancy in annotated sequences and a lower number of unannotated sequences as well as poor sampling of single-copy sequences. Based on the number of annotated contigs our results for IGA analysis are similar to $\mathrm{Lu}$ et al. [22]. Considering the number of assembled nucleotides in contrast to the number of contigs, the present two assemblies were quite comparable, $70 \%$ in the IGA transcriptome assembly vs. $82 \%$ in the Sanger-EST assembly. In the Sanger-EST assembly 23\% of the contigs or $17.5 \%$ of nucleotides did not align to any homologous sequences in the GenBank, therefore these sequences can be identified as potential novel transcripts or genes in pepper that were not previously characterized or simply were too short for conclusive annotation. Not surprising, the annotations of both assemblies presented here are very similar in terms of species distribution of top-hits. This is probably due to the bias in databases toward having more data for certain species that have been annotated better than the others. At the time of analysis tomato genome annotation was not available in GenBank databases which could be the reason as to why $S$. lycopersicum is not on the top of species hit list.

Another aspect of our study was to assign transcripts to different metabolic pathways. Generating KEGG maps 
and designating enzymes to different metabolic pathways is an effective way to identify candidate genes. In an ultra-saturated genetic map of pepper, contigs that are spanning a QTL can be further examined for their role in one or more metabolic pathways. Finding annotated contigs will then help to identify KEGG maps related to the enzymes and metabolites involved in the traits and further investigate their function in controlling traits.

One of our goals in this project was to develop markers that can readily be used in breeding programs. We presented here two sets of markers, SSR and SNP for genetic and breeding analyses in pepper. The putative SNPs that were discovered in the Sanger-EST assembly were internally validated by KASPar assays in a genotyping panel of 43 pepper lines and accessions. It is deemed to be very robust and reliable despite the lower sequence depth compared to SNPs that were discovered in the IGA transcriptome assembly. We also observed a comparable SNP frequency in both assemblies $(1 / 2,798 \mathrm{bp}$ vs.1/2,523 bp) indicating SNP frequency in pepper transcriptomes is plausibly consistent across methods and accessions used in different experiments. Coincidently, the polymorphism among three diverse lines, CM334, Early Jalapeño and Maor [11], and those within the $\mathrm{F}_{1}$-hybrid of Bukang was similar.

\section{Conclusions}

There was a great need to generate an abundant number of molecular markers for breeding programs of pepper. To that end, assembling transcriptomes seemed very promising in the identification of thousands of highquality markers before a pepper genome sequence becomes available. As a result of our efforts, the generated markers are currently being used in genetic mapping and QTL analyses by different groups around the globe. In order to have a better understanding of the assembled sequences and to identify candidate genes underlying QTLs, we also annotated the contigs of Sanger-EST and RNAseq assemblies. These and other information have been curated in a database that we have dedicated for pepper GeneChip project (see Data Access). Nevertheless, the main task still will remain to sequence the pepper genome and to use the available genetic resources to develop new pepper varieties with higher yields, better flavors and more resistance to biotic as well as abiotic stresses.

\section{Data access}

The raw data are publically available through The NCBI Sequence Read Archive (SRA) under accession No. SRA052314.2 and the trimmed reads submitted to The European Nucleotide Archive (ENA) under study number ERP001411. The SNPs were submitted to database of Single Nucleotide Polymorphisms (dbSNP). Bethesda
(MD): National Center for Biotechnology Information, National Library of Medicine. dbSNP accession: [ss523750580 - ss524344808], (dbSNP Build ID: B138), available from the NCBI SNP database website on the next build in December 2012. The IGA transcriptome assembly was submitted to NCBI transcriptome shotgun assembly database (TSA) under BioProject No. PRJNA163071 and TSA accession numbers JW05245 - JW111875. Both assemblies, annotations, SNPs, SSRs and other information are also available at https://pepper.ucdavis.edu/public/ data.php.

\section{Methods}

\section{Assembly of pepper Sanger-EST sequences}

\section{Source of Sanger-EST assembly sequences}

Pepper sequences were obtained from two sources. A total of 115,787 EST sequences from 21 cDNA libraries (Korean GenePool database) of an $\mathrm{F}_{1}$ hybrid variety, Bukang, were kindly provided by Dr. Doil Choi (Korean Research Institute of Bioscience \& Biotechnology (KRIBB), now at Seoul National University) [22]. These sequences were combined with other sequences from GenBank (in 2006), trimmed and passed through quality assessments to be used in assembly (see below). Tissue collection and cDNA library construction and Sanger sequencing has been described elsewhere [22]. GenBank Sequences (in 2006) included ESTs (31,495), mRNAs (515) and genomic sequences (464). Of these 21,590 were from KRIBB.

\section{Preparation of sequences for assembly}

To remove the redundant EST sequences from the GenBank collection, any sequence with an identical ID to the KRIBB collection was removed to obtain a nonredundant set of sequences. Genbank mRNA sequences were directly used for assembly. We identified two types of genomic sequences from the GenBank collection, annotated (218) and unannotated (246) sequences. The exon and intron regions of annotated sequences were known. Therefore we simply split out the introns to obtain the exonic sequences. In the case of unannotated sequences, the basic local alignment tool (BLASTX cutoff value $=1 \mathrm{e}^{-20}$ ) was used to search against plant reference genes to extract coding regions. The KRIBB sequences were merged with the processed GenBank sequences in the next step. The merged data set was further checked for regions containing low complexity sequences or vector sequences using custom made Python, TCL and Perl scripts that can be accessed from "atgc tools" website.

\section{Clustering and assembly of pepper Sanger-EST sequences}

CAP3 software [29] was used for assembling the sequences with overlap length cutoff of 100 and overlap 
percent identity cutoff of 90. Visualization and analysis of DNA sequences alignments generated by CAP3 (to detect and validate polymorphic sites either SNPs or InDels) was carried out using custom made "atgc-tools/ align" scripts. These alignments and consensus sequences can be accessed through the pepper GeneChip website.

\section{Assembly of pepper IGA transcriptomes reads Plant materials and library construction}

The seed of three pepper (C. annuum) lines 'CM334,' 'Maor' and 'Early Jalapeño' (EJ) were planted in the greenhouses of the Department of Plant Sciences at UC Davis under standard conditions for Capsicum [30] until adult stage. Three cDNA libraries (one from each pepper variety) were prepared using pooled mRNA that was independently extracted from seven tissues: root, young leaf, 5, 10, and 20 days post pollination developing fruit, breaker and ripe fruit using Qiagen RNeasy Mini Kit (Qiagen Valencia CA, USA) per the manufacture's protocol. CM334 root tissue was inoculated with Phytophthora capsici to induce expression of resistance genes. Aliquots were quantified using a NanoDrop spectrophotometer (NanoDrop Wilmington, USA) and checked for quality by electrophoreses separation using Lonza FlashGel System FlashGel RNA Cassettes (Lonza Inc. Allendale, USA). Samples were pooled in equivalent concentration. For each pepper line, paired-end libraries were prepared following standard Illumina protocols (Paired-End DNA Sample Prep Kit). The libraries were sheared and 300-350 bp fragments were selected on gels. The libraries were normalized using doublestranded nuclease to digest high copy double--stranded DNA during re--association after denaturation and then prepared for sequencing as described by Illumina [31]. The cDNA libraries were sequenced using Illumina Genome Analyzer II (IGA) for 85 cycles per direction at the UC Davis Genome Center. One lane of paired-end pass and one lane of single pass were run for each of CM334 and Maor lines and two lanes of paired-end pass were run for Early Jalapeño.

\section{De novo assembly of IGA reads}

The IGA data went through our standard preprocessing pipeline, developed at UCD (ILLUPA, A. Kozik, Pers. comm.). The trimming stringency was based on a study that was carried out by Alex Kozik to trim Illumina short reads of lettuce [32]. The reads (sequences) were first trimmed to discard traces of adapters and primers that were added to cDNA during library preparation using "cutadapt" software. Under the normal trimming scheme we trimmed the 5' and 3' ends of the reads with quality scores of lower than 20 (or 0.01 probability of error), then we retained the reads between a minimum length of $40 \mathrm{nt}$ and a maximum of $85 \mathrm{nt}$ with no further trimming (full filtered length). Under a more stringent procedure we trimmed the full filtered length reads (from above 40-85 nt) more robustly by trimming $10 \mathrm{nt}$ from 5' end and 5 nt from 3' end of each read (25-70 nt length). As a result we maintained the reads with a length between $25 \mathrm{nt}$ and $70 \mathrm{nt}$.

Velvet (v 1.0.14) [20] and CLC Genomics Workbench (v 4.0.3) software packages were used to assemble the sequences. For each pepper genotype, a Velvet assembly with several $k$-mers (31, 35 and 41 Velvet hash setting) was performed using full length trimmed and 25-70 nt length trimmed data. DNA K-mer is synonymous to a word in our language. It is a short consecutive stretch of DNA that will be used in de bruijn graph as described elsewhere [20].

The results of all $k$-mer assemblies were combined with CAP3 to make a line-specific super assembly. In other words, for each pepper line we obtained six Velvet assemblies (3 k-mers settings by 2 sets of reads) that were combined with CAP3 software yielding a super assembly. In addition to Velvet assemblies, two iterations of assembly (one for normally trimmed reads and one for stringently trimmed reads) with CLC genomic workbench with default settings (Insertion/deletion cost $=3$, mismatch cost $=2,80 \%$ of read length with similarity of 90\%) were carried out for each pepper genotype. The results of the combined Velvet assemblies (super assembly) and CLC assemblies were merged using CAP3 software to make the Mega assembly for each line. Once we generated three Mega assemblies (one per pepper genotype), we combined the Mega assemblies from each line by CAP3 software to obtain a pepper transcriptome Meta (IGA) assembly. A graphical presentation of the assembly procedure is depicted in [See Additional File 3: Figure S16]. The IGA transcriptome assembly was submitted to NCBI transcriptome shotgun assembly database (TSA) under BioProject No. PRJNA163071 and TSA accession numbers JW05245 - JW111875.

\section{GO annotation of the Sanger-EST and the IGA assemblies}

The Blast2GO [26] software was used to annotate both assemblies. Blast2GO involves three main steps, 1) BLASTX of the nucleotide sequence against the nonredundant protein database (nr) of NCBI, 2) mapping, retrieving GO terms associated with the blast results, and 3) annotating GO terms associated with each query in order to relate the sequences to known protein function. Briefly, a BLASTX search of contig nucleotide sequences against the non-redundant protein database (nr) of NCBI was performed under the default settings of BLAST2GO and the BLAST expectation value of $1.0 \mathrm{e}^{-3}$ and maximum 20 hits, HSP length cutoff (default = 33) with low complexity filter on was used. The GO 
terms associated with each BLAST hit were retrieved (mapping step) and GO annotation assignment (annotation step) to the query sequences was carried out using the following annotation score parameters; E-Value Hit Filter (default=1.0E-6), Annotation Cut-Off (default $=55$ ), GO-Weight (default=5), Hsp-Hit Coverage Cut Off (default $=0$ ). In addition, contig sequences were queried for conserved domains/motifs using InterProScan, an on-line sequence search plug-in within the BLAST2GO program with all 13 applications selected before run and the resulting GO terms were merged with the GO term results from the annotation step of Blast2GO. KEGG maps for more than 130 metabolic pathways were generated with the KEGG extension of Blast2GO.

\section{Identification of SNPs in the Sanger-EST and the IGA assemblies \\ Sanger-EST assembly SNPs}

In order to discover putative SNPs in the Sanger-EST assembly, the output files of CAP3 were used in the pipeline of SNP discovery (Alex Kozik, Pers. Comm.). In this method only contigs that are the results of assembling a minimum of two ESTs can be interrogated for the existence of putative SNPs. A total of 18,226 unigenes in the Sanger-EST assembly were singletons. As a result only 12,970 out of 31,196 unigenes were surveyed for SNPs. In Kozik's pipeline, the EST sequences first align (map) to their corresponding consensus sequences. Second, at each position of consensus sequence the program searches the pileup of EST sequences for base changes among sequences. In the last step, the program outputs a list of contigs and positions where differences were found. A separate filtering step was carried out by a Perl script to select the SNPs with minimum depth of 2 for each SNP allele, 50 bp from the start or the end of a contig. If the two SNPs were in the vicinity of $50 \mathrm{bp}$ from each other only the one with higher coverage was selected.

\section{IGA transcriptome assembly SNPs}

Assembling transcriptomes of three pepper lines enabled us to map all the IGA reads back to the assembly and to determine the putative SNPs. BWA [33], SAMtools, and in-house Perl scripts were used to call the SNPs. First we mapped all the short reads of each line separately to the assembly using BWA aligner to generate 3 BAM files. Using the SAMtools pileup command the variable positions (SNPs) were determined between the consensus pepper assembly and each line. The BAM files were also merged by SAMtools [34] and polymorphism were determined between the merged files and assembly. Custom written Perl scripts were used to generate a genotype table where we could line up the consensus assembly with genotype call for all three pepper lines. A position was called a putative SNP if two out of three pepper accessions/lines had the same homozygous allele (minimum depth of 10 reads), but different from the third homozygous accession. For instance, if CM334 and Maor were rendering a $\mathrm{G}$ allele at a given position and Early Jalapeño was carrying a $\mathrm{C}$ allele at the same position, then the position was called a putative SNP. In cases where the position of a SNP could not be unequivocally determined as described above then that position was called a heterozygote. The putative SNPs were then filtered against intron-exon junction positions using the command line version of Intron Finder software at Sol Genomics Network (SGN) website. The filtered putative SNPs were set to be at least $50 \mathrm{bp}$ from intron/exon splice junctions as well as adjacent SNPs and heterozygote positions.

\section{Validation of SNPs in the Sanger-EST assembly}

In order to validate the in silico SNPs from the SangerEST assembly, 50 nucleotides from either side of 142 SNPs, (40 of which corresponded to SPP markers from a Pepper diversity panel [11]) were extracted from each contig. Sequences were sent to KBiosciences to develop KASPar assays. The assays were run by KBioscience on a diversity panel of 47 lines and cultivars and the data was visualized by KBioscience SNP viewer software and further analyzed with Microsoft Excel.

\section{Validation of SNPs in the IGA transcriptome assembly}

The three pepper lines that were used for the IGA transcriptome assembly were also included in the genotyping panel that was surveyed for SNPs by KASPar assay. We used BLASTN to find near identical sequences of the IGA transcriptome assembly to 101 bases flanking each SNP (50 nt each side) that was used in the KASPar assay. If a hit was found with $95 \%$ sequence similarity and $\mathrm{e}^{-20}$ expectation value, then we investigated the possibility of calling the same SNP in the IGA transcriptome assembly by scanning the list of IGA transcriptome based SNPs.

\section{In silico identification of SSRs in Sanger-EST and IGA transcriptome assemblies}

The assembled sequences were used to identify signatures of SSRs. FASTA files containing all the assembled sequences were used as an input file in MISA Perl script to specify the minimum number of the following repeats for microsatellites (unit size/minimum number of repeats): (2/6) (3/5) (4/5) (5/5) (6/5). MISA has the capability of predicting perfect (SSRs with no interruption) and compound SSRs (SSRs with a spacer sequence). The variable to specify the maximum length of the spacer sequence was set as 100bp in the MISA setup file. 


\section{Primer design for SSRs}

The PRIMER3 software [35] was used to design forward and reverse primers flanking the SSR containing sequence. An accompanying Perl script of MISA software (p3_in.pl) was used to make the input file for PRIMER3. A second accompanying Perl script of MISA software (p3_out.pl) was used to parse the output file of PRIMER3 into a user friendly output. The target amplicon size was set as $100-300 \mathrm{bp}$, with optimal annealing primer temperature of $60{ }^{\circ} \mathrm{C}$ and optimal primer length as 20 nucleotides.

\section{Additional files}

\section{Additional file 1: Ashrafi et al. 2012 Pepper Annotation}

Supp 05072012. A Microsoft-Word 2007 file with 16 figures comparing the results of Blast2GO for GeneChip (Sanger-EST) and transcriptome assemblies of pepper as well as the IGA transcriptome assembly procedure flow chart.

Additional file 2: Ashrafi et al. Pepper Assembly Supp 05072012. A Microsoft-Excel 2007 file with 2 tables (worksheet) corresponding to Annotation results of the Sanger-EST and IGA transcriptome assemblies of pepper.

Additional file 3: Ashrafi et al. Pepper Assembly Supp 05072012. A Microsoft-Excel 2007 file with 13 tables corresponding to SSR and SNP lists identified in both the Sanger-EST and IGA transcriptome assemblies of pepper. It also includes identified SSR motifs and list of diversity panel (adapted from Hill et al. [11]).

\section{Abbreviations and URLs}

454 Life Sciences: http://www.454.com

ABySS: http://www.bcgsc.ca/platform/bioinfo/software/abyss;

Alexander Kozik SNP discovery pipeline: http://cgpdb.ucdavis.edu/

SNP_Discovery_CDS/;

Alexander Kozik Illupa pipeline: http://code.google.com/p/atgc-illumina/ downloads/list:

atgc-Tool website: http://code.google.com/p/atgc-tools/;

atgc-Tool/align website: http://code.google.com/p/atgc-align/;

CAP3 Software: http://pbil.univ-lyon1.fr/cap3.php;

CLC Genomics Workbench: http://www.clcbio.com/;

Cutadapt software: http://code.google.com/p/cutadapt/;

European Nucleotide Archive (ENA): http://www.ebi.ac.uk/ena/;

GenBank (NCBI): http://www.ncbi.nlm.nih.gov/;

Illumina GoldenGate Assay/Illumina Technology: http://www.llumina.com/;

InterProScan: www.ebi.ac.uk/Tools/pfa/iprscan/;

KBioscience: http://www.kbioscience.co.uk/

KBioscience SNP Viewer Software: http://www.kbioscience.co.uk/software/

Software_intro.html;

KEGG Maps: http://www.genome.jp/kegg/pathway.html;

Korean GenePool database: http://genepool.kribb.re.kr;

Korea Research Institute of Bioscience and Biotechnology: http://www.kribb.

re.kr/eng/;

The NCBI Sequence Read Archive (SRA): http://www.ncbi.nlm.nih.gov/Traces/sra;

The NCBI SNP data base: http://www.ncbi.nlm.nih.gov/SNP/;

MISA Perl Script: http://pgrc.ipk-gatersleben.de/misa/;

Newbler: http://454.com/products/analysis-software/index.asp;

Pepper GeneChip Website: https://www.pepper.ucdavis.edu/;

Sequenom: http://www.sequenom.com/:

SOAPdenovo: http://soap.genomics.org.cn/;

SOLiD: http://www.appliedbiosystems.com/absite/us/en/home/applications-

technologies/solid-next-generation-sequencing.html;

Sol Genomics Network: http://solgenomics.net/;

Transcriptome shotgun assembly database (TSA): http://www.ncbi.nlm.nih.gov/ genbank/tsa;

Velvet Sequence Assembler: http://www.ebi.ac.uk/ zerbino/Velvet/.

\section{Competing interests}

This project was supported by an award from the University of California UC Discovery Program bio06-10565 with matching funds from Rijk Zwaan BV Enza Zaden BV, DeRuiter Seeds B.V., Nunhems USA, Seminis Vegetable Seeds, Inc., Syngenta Seeds, Inc. and Vilmorin Co. The funders had no role in study design, data collection and analysis, decision to publish, or preparation of the manuscript. Funds were provided directly to the University of California Davis. Authors have no competing interests for this research.

\section{Authors' contributions}

HA wrote the manuscript and performed IGA transcriptome assembly, SNP and SSR discovery. TAH, planted peppers, collected tissues, prepared DNA for KASPar assay and analyzed the KASPar assay data, read and edited the manuscript. KS, developed the mRNA library for IGA sequencing. SCR, developed SNP discovery pipeline in the transcriptome and designed KASpar assays. AK, co-designed the project and developed the SNP detection pipeline of EST assembly. JY, processed and assembled Sanger ESTs sequences. AVD, envisioned, realized the project and supervised the group. All authors read and edited the manuscript. All authors read and approved the final manuscript.

\section{Acknowledgments}

This study was financially supported by De Ruiter Seeds, Enza Zaden, Seminis Vegetable Seeds, Nunhems, Rijk Zwaan, Syngenta, Vilmorin and UC Discovery program. The authors would like to thank Dr. Doil Choi, Seoul National University, who provided Sanger-EST sequences. We also would like to thank the sequencing facility of UC Davis Genome Center and Bioinformatics core facility to provide us the servers and computational power. The assembly with CLC and annotation of the assemblies would not be possible without collaboration with Drs. Dean Lavelle and Marta Matvienko (former member) of Dr. Richard Michelmore's laboratory.

\section{Author details}

'Seed Biotechnology Center, University of California, Davis, 1 Shields Ave, Davis, CA 95616, USA. ${ }^{2}$ Genome Center, University of California, Davis, 1 Shields Ave, Davis, CA 95616, USA. ${ }^{3}$ Present address: Interdisciplinary Center for Biotechnology Research, University of Florida, Gainesville, FL 32610, USA.

Received: 8 May 2012 Accepted: 22 October 2012

Published: 30 October 2012

\section{References}

1. Bosland PW, Votova EJ: Peppers: Vegtable and spice capsicums, Volume 12. New York: CABl; 2000.

2. Paran I, van der Voort JR, Lefebvre $V$, Jahn $M$, Landry L, van Schriek $M$ Tanyolac B, Caranta C, Chaim AB, Livingstone K, et al: An integrated genetic linkage map of pepper (Capsicum spp.). Mol Breed 2004, 13(3):251-261

3. Lefebvre V, Pflieger $S$, Thabuis A, Caranta C, Blattes A, Chauvet J-C, Daubèze A-M, Palloix A: Towards the saturation of the pepper linkage map by alignment of three intraspecific maps including known-function genes. Genome 2002, 45:839-854

4. Wu F, Eannetta N, Xu Y, Durrett R, Mazourek M, Jahn M, Tanksley S: A COSII genetic map of the pepper genome provides a detailed picture of synteny with tomato and new insights into recent chromosome evolution in the genus Capsicum. TAG Theor Appl Genet 2009, 118(7):1279-1293.

5. Rafalski A: Applications of single nucleotide polymorphisms in crop genetics. Curr Opin Plant Biol 2002, 5:94-100.

6. Arumuganathan K, Earle ED: Nuclear DNA content of some important plant species. Plant Mol Biol Rep 1991, 9(3):208-218.

7. Van Deynze A, Stoffel K, Buell CR, Kozik A, Liu J, van der Knaap E, Francis D: Diversity in conserved genes in tomato. BMC Genomics 2007, 8:465.

8. Van Deynze A, Stoffel K, Lee M, Wilkins TA, Kozik A, Cantrell RG, Yu JZ, Kohel RJ, Stelly DM: Sampling nucleotide diversity in cotton. BMC Plant Biol 2009, 9:125

9. Ganal MW, Altmann T, Röder MS: SNP identification in crop plants. Curr Opin Plant Biol 2009, 12(2):211-217.

10. Labate JA, Baldo AM: Tomato SNP discovery by EST mining and resequencing. Mol Breed 2005, 16(4):343-349. 
11. Hill T, HA H, Chin-Wo SR JJY, Stoffel K, Truco M-J, Kozik A, Michelmore RW, Deynze AV: Development of a pepper Genechip array and its application for marker discovery and analysis of Capsicum germplasm diversity. PLOS One 2012, In Press.

12. Stoffel K, Leeuwen H, Kozik A, Caldwell D, Ashrafi H, Cui X, Tan X, Hill T, Reyes-Chin-Wo S, Truco M-J, et al: Development and application of a 6.5 Million feature Affymetrix GeneChip(R) for massively parallel discovery of single position polymorphisms in lettuce (Lactuca spp.). BMC Genomics 2012, In press.

13. Barbazuk WB, Emrich SJ, Chen HD, Li L, Schnable PS: SNP discovery via 454 transcriptome sequencing. Plant J 2007, 51(5):910-918.

14. Trick M, Long $Y$, Meng J, Bancroft I: Single nucleotide polymorphism (SNP) discovery in the polyploid Brassica napus using Solexa transcriptome sequencing. Plant Biotechnol J 2009, 7(4):334-346.

15. Stratton M: Genome resequencing and genetic variation. Nat Biotech 2008, 26(1):65-66.

16. Lister R: Highly integrated single-base resolution maps of the epigenome in Arabidopsis. Cell 2008, 133:523-536.

17. Zhang G, Guo G, Hu X, Zhang Y, Li Q, Li R, Zhuang R, Lu Z, He Z, Fang X, et al: Deep RNA sequencing at single base-pair resolution reveals high complexity of the rice transcriptome. Genome Res 2010, 20(5):646-654.

18. Mortazavi A, Williams BA, McCue K, Schaeffer L, Wold B: Mapping and quantifying mammalian transcriptomes by RNA-Seq. Nat Meth 2008 5(7):621-628.

19. Rowley JW, Oler AJ, Tolley ND, Hunter BN, Low EN, Nix DA, Yost CC, Zimmerman GA, Weyrich AS: Genome-wide RNA-seq analysis of human and mouse platelet transcriptomes. Blood 2011, 118(14):e101-e111.

20. Zerbino D, Birney E: Velvet: algorithms for de novo short read assembly using de Bruijn graphs. Genome Res 2008, 18:821-829.

21. Simpson JT, Wong K, Jackman SD, Schein JE, Jones SJM, Birol I: ABySS: a parallel assembler for short read sequence data. Genome Res 2009, 19(6):1117-1123.

22. Lu F-H, Cho M-C, Park Y-J: Transcriptome profiling and molecular marker discovery in red pepper, Capsicum annuum L. TF68. Mol Biol Rep 2012, 39(3):3327-3335

23. Lu F-H, Yoon M-Y, Cho Y-I, Chung J-W, Kim K-T, Cho M-C, Cheong S-R, Park $Y$-J: Transcriptome analysis and SNP/SSR marker information of red pepper variety YCM334 and Taean. Sci Hortic 2011, 129(1):38-45.

24. Góngora-Castillo E, Fajardo-Jaime R, Fernández-Cortes A, Garfias AEJ, Lozoya-Gloria E, Martínez O, Ochoa-Alejo N, Rivera-Bustamante R: The capsicum transcriptome DB: a "hot" tool for genomic research. Bioinformation 2012, 8(1):043-047.

25. Ashburner M, Ball CA, Blake JA, Botstein D, Butler H, Cherry JM, Davis AP, Dolinski K, Dwight SS, Eppig JT, et al: Gene Ontology: tool for the unification of biology. Nat Genet 2000, 25(1):25-29.

26. Conesa A, Götz S, García-Gómez JM, Terol J, Talón M, Robles M: Blast2GO: a universal tool for annotation, visualization and analysis in functional genomics research. Bioinformatics 2005, 21(18):3674-3676.

27. Hill T, Ashrafi H, Reyes-Chin-Wo S, Solano Romero M, Van Deynze A, Kozik A Comparisons of high-density EST-based maps in pepper species. In Plant Biology. Minneapolis, MN: American Society of Plant Biologists; 2011:15037. Aug. 6-10

28. Ruepp A, Zollner A, Maier D, Albermann K, Hani J, Mokrejs M, Tetko I, Güldener U, Mannhaupt G, Münsterkötter M, et al: The FunCat, a functional annotation scheme for systematic classification of proteins from whole genomes. Nucleic Acids Res 2004, 32(18):5539-5545.

29. Huang $X$, Madan A: CAP3: A DNA sequence assembly program. Genome Res 1999, 9:868-877.

30. Bosland PW, Lindsey DL: A seedling screen for Phytophthora root rot of pepper, Capsicum annuum. Plant Dis 1991, 75(10):1048-1050.

31. mRNA sequencing sample preparation guide. 24. http://grcf.jhmi.edu/hts/protocols/mRNA-Seq_SamplePrep_1004898_D. pdf.

32. Illumina Pipeline and Assembly, a case study in lettuce. http://atgc-illumina.googlecode.com/files/ ILLUPA_Overview_AKozik_100108_A.pdf

33. Li H, Durbin R: Fast and accurate short read alignment with Burrows-Wheeler transform. Bioinformatics 2009, 25(14):1754-1760.
34. Li H, Handsaker B, Wysoker A, Fennell T, Ruan J, Homer N, Marth G, Abecasis G, Durbin R, Subgroup GPDP: The sequence alignment/map format and SAMtools. Bioinformatics 2009, 25(16):2078-2079.

35. Rozen S, Skaletsky H: Primer 3 on the www for general users and for biologist programmers. Methods Mol Biol 2000, 132:365-386.

doi:10.1186/1471-2164-13-571

Cite this article as: Ashrafi et al:: De novo assembly of the pepper transcriptome (Capsicum annuum): a benchmark for in silico discovery of SNPs, SSRs and candidate genes. BMC Genomics 2012 13:571.

\section{Submit your next manuscript to BioMed Central and take full advantage of:}

- Convenient online submission

- Thorough peer review

- No space constraints or color figure charges

- Immediate publication on acceptance

- Inclusion in PubMed, CAS, Scopus and Google Scholar

- Research which is freely available for redistribution

Submit your manuscript at www.biomedcentral.com/submit
C) BioMed Central 